\title{
MYH9 is crucial for stem cell-like properties in non-small cell lung cancer by activating mTOR signaling
}

\author{
Meng Chen ${ }^{1}$, Li-Xin Sun ${ }^{1}$, Long Yu ${ }^{1}$, Jun Liu ${ }^{1}$, Li-Chao Sun ${ }^{1}$, Zhi-Hua Yang ${ }^{1}$, Xiong Shu $\mathbb{D}^{2 凶}$ and Yu-Liang Ran (D) ${ }^{1 凶}$
}

(c) The Author(s) 2021

The fatality rate of non-small cell lung cancer (NSCLC) has been high due to the existence of cancer stem cells (CSCs). Non-muscle myosin heavy chain 9 (MYH9) can promote the progression of various tumors, but its effect on the stem cell-like characteristics of lung cancer cells (LCCs) has not been clarified. Our research found that the stemness characteristics of LCCs were significantly enhanced by the overexpression of MYH9, and the knockout of MYH9 had the opposite effects. The in vivo with inhibitor blebbistatin further confirmed the effect of MYH9 on the stem cell-like behavior of LCCs. Furthermore, western blotting showed that the expression level of CSCs markers (CD44, SOX2, Nanog, CD133, and OCT4) was also regulated by MYH9. Mechanistic studies have shown that MYH9 regulates stem cell-like features of LCCs by regulating the mTOR signaling pathway, which was supported by sphere formation experiments after LCCs were treated with inhibitors Rapamycin and CHIR-99021. Importantly, high expression of MYH9 in lung cancer is positively correlated with poor clinical prognosis and is an independent risk factor for patients with NSCLC.

Cell Death Discovery (2021)7:282; https://doi.org/10.1038/s41420-021-00681-z

\section{INTRODUCTION}

Lung cancer has always been considered a serious disease characterized by poor prognosis and a low survival rate. The morbidity and mortality of lung cancer remain high, which is closely related to the prevalence of tobacco and the deterioration of the environment $[1,2]$. Despite the continuous progress in diagnosis and treatment, the number of deaths from lung cancer is still increasing, mainly caused by the recurrence, metastasis, and drug resistance of lung cancer.

The occurrence and maintenance of lung cancer depend on the presence of lung cancer stem cells (LCSCs), which contribute to the poor prognosis of patients with lung cancer [3-6]. In 2005, Kim et al. found that the bronchiolar alveolar stem cells isolated from the lung injury model were the source cells of lung adenocarcinoma [7]. In 2007, Ho et al. isolated side population cells with strong tumorigenicity, self-renewal ability, invasion ability, and drug resistance from LCCs, and proved that these cells are the initial cells of lung cancer [8]. Subsequently, researchers found CD133, CD44, and other LCSC surface markers and related signal pathways [9-13], further confirming the existence of LCSCs. At present, various targeted drugs for cancer stem cells (CSCs) have entered phase I or phase II clinical trials [14-16], which is expected to bring a breakthrough in cancer therapy. However, because of the complexity of the structure and diversity of lung tumor tissue subtypes, it is difficult to identify specific targets for LCSCs. Thus, the new therapeutic targets for LCSCs have become an urgent goal in lung cancer treatment.
As a skeleton-related protein, non-muscle myosin heavy chain 9 (MYH9) is widely expressed in cells and tissues and belongs to a kind of nonmuscle myosin II (non-muscle myosin, NM II) [17]. MYH9 can hydrolyze ATP, convert chemical energy into mechanical motion and participate in various processes that require contractile force, such as cell division, migration, shape maintenance, and signal transduction [18, 19]. MYH9 mutations lead to autosomal-dominant diseases, kidney diseases, and thrombotic diseases [20-23] and participate in the occurrence and development of a variety of tumors. The researchers found that the highly expressed $\mathrm{MYH} 9$ was involved in the metastasis and recurrence of gastric, colorectal, renal, esophageal, and breast cancer [24-28]. The high expression of MYH9 in NSCLC was found by individual researchers [29]. Some data also show that MYH9 can affect the maintenance characteristics of mesenchymal stem cells and gastrointestinal epithelial stem cells [30, 31]. However, research on LCSCs is lacking, researchers know little about its function in the process of NSCLC, the specific molecular and related signal pathway regulation mechanisms have not been clarified.

In the research, we analyzed the stemness regulatory function of MYH9 on LCCs and its mechanism, and found that MYH9 enhanced the stem cell-like biological behavior of LCCs by activating the mTOR signaling pathway. Also, we used an immunohistochemical (IHC) assay to analyze the expression of MYH9 in clinical tissue samples of lung cancer and found that it can be used as an independent factor in evaluating the prognosis of patients with NSCLC.

\footnotetext{
${ }^{1}$ State Key Laboratory of Molecular Oncology, National Cancer Center/National Clinical Research Center for Cancer/Cancer Hospital, Chinese Academy of Medical Sciences and Peking Union Medical College, Beijing, China. ${ }^{2}$ Beijing Research Institute of Orthopaedics and Traumatology, Beijing JiShuiTan Hospital, Beijing, China. 然mail: shuxiong654321@aliyun.com; ran_yuliang@126.com
} 


\section{RESULTS}

Enrichment and expression of MYH9 in spheroid cells of lung cancer

To study the expression and localization of MYH9 in NSCLC cell lines, we performed a live-cell and fixed-cell immunofluorescence assay used in six cell lines, namely $\mathrm{NCl}-\mathrm{H} 460$, SPCA-1, A549, NCl-H1299, NCl-H226, and GLC-82. MYH9 was expressed in the cytoplasm, membrane, and nucleus of different LCCs (Fig. $1 \mathrm{~A})$. Serum-free enrichment culture in vitro is a simple and reliable method for screening CSCs. The results showed that the spheroid formed by $\mathrm{A} 549, \mathrm{NCl}-\mathrm{H} 460$, and $\mathrm{NCl}-\mathrm{H} 226$ exhibited a tightened appearance (Fig. 1B). Subsequently, the expression of MYH9 from parent and spheroid cells in different LCCS was studied by flow fluorescence assay. Results (Fig. 1C) showed that, the proportion of MYH9 in spheroid cells from $\mathrm{NCl}-\mathrm{H} 226$, $\mathrm{NCl}-\mathrm{H} 460$, and $\mathrm{A} 549$ were $12.4 \%, 10.5 \%$, and $8.49 \%$, respectively, whereas in the parent cells were $4.92 \%, 3.48 \%$, and $2.77 \%$, respectively. The positive expression rate of spheroid cells was $2.5,3.0$, and 3.1 times that of parent cells, respectively. The expression of MYH9 in spheroid cells from $\mathrm{NCl}-\mathrm{H} 226, \mathrm{NCl}-\mathrm{H} 460$,
A

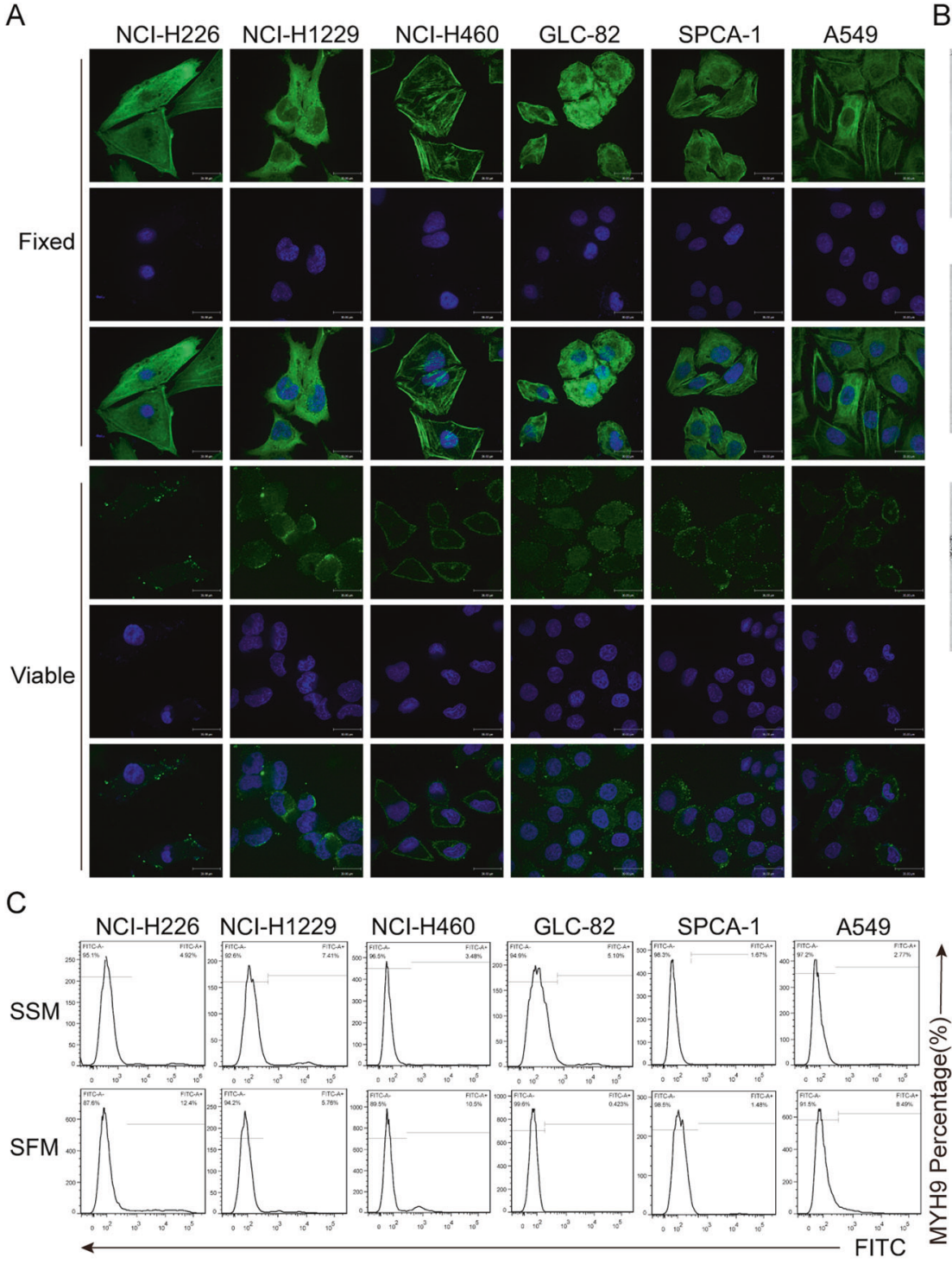

D

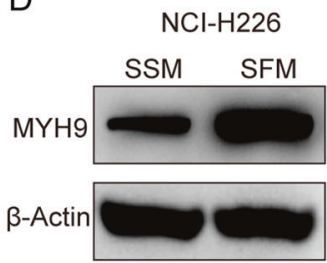

$\mathrm{NCl}-\mathrm{H} 1229$
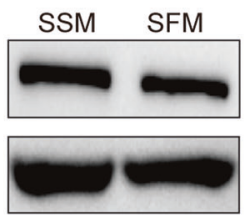

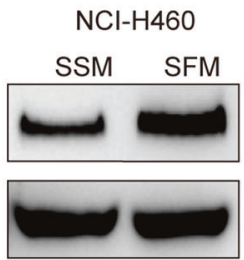

GLC-82

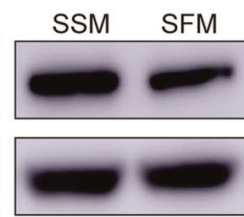

B

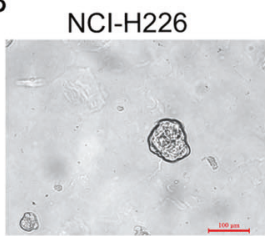

$\mathrm{NCl}-\mathrm{H} 1229$
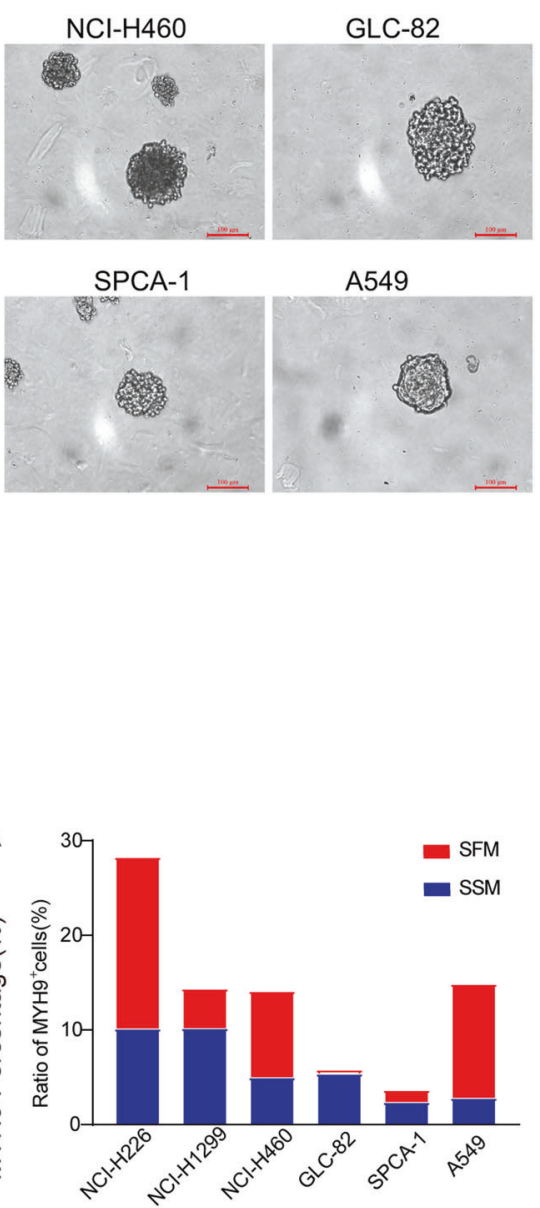

SPCA-1

A549
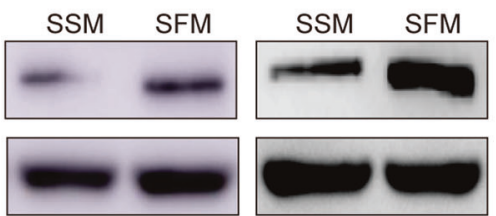

Fig. 1 Enrichment and expression of MYH9 in spheroid cells of lung cancer. A Expression of MYH9 in lung cancer cell lines (GLC-82, SPCA-1, $\mathrm{NCl}-\mathrm{H} 460, \mathrm{~A} 549, \mathrm{NCl}-\mathrm{H} 226$, and NCl-H1299) (scale bar, $30 \mu \mathrm{m}$ ). B Serum-free suspension culture of LCCs. C Analysis of the proportion of MYH9positive cells in parent and spheroid cells of lung cancer by flow fluorescence. D The expression level of MYH9 in parent and spheroid cells of lung cancer cell lines. Data are expressed as mean \pm SEM of three independent experiments. ${ }^{*} p<0.05,{ }^{* *} p<0.01$, compared to SSM. 
and A549 was upregulated (Fig. 1D) compared with parent cells. These results suggest that MYH9 is expressed in the membrane, cytoplasm, and nucleus of LCCs and is enriched in spheroid cells, which may identify LCSCs.

\section{Downregulation of MYH9 expression suppresses the stem cell- like malignant phenotype of LCCs in vivo and in vitro}

To study the effect of MYH9 on biological behavior related to the stemness of LCCs, we selected $\mathrm{NCl}-\mathrm{H} 460$ with high endogenous expression of MYH9 to construct the knockdown stable transformation cell line (Fig. 2A). The clone formation assay is used to detect the regulatory role of MYH9 on the proliferation of LCCs. The results showed that the proliferation ability of MYH9 knockdown cells decreased significantly in vitro (Fig. 2B, C). To explore the sphere-forming ability of MYH9 knockdown cells, we found that the expression of MYH9 was down-regulated, the number of sphere-forming cells decreased significantly, and the inhibition rate of sphere-forming ability reached $86 \%$ in $\mathrm{NCl}-\mathrm{H} 460-$ shMYH9 cells (Fig. 2D). Compared with the control cells, the protein expression levels of CD44, CD133, SOX2, Nanog, and OCT4 in MYH9 knockdown cells were decreased (Fig. 2E), indicating that MYH9 affected the expression of stemness-related genes.

The ability of invasion and migration plays an important role in the metastasis and recurrence of tumor. The migration and invasion ability of MYH9 knockdown cells was significantly weaker than that of control cells (Fig. 2F). Western blot also detected the expression of EMT marker in MYH9 knockdown cells. After the downregulation of MYH9, E-cadherin was significantly upregulated, whereas the expression of $\mathrm{N}$-cadherin, Vimentin, and Snail was significantly downregulated (Fig. 2G), indicating that MYH9 promotes the invasion and migration of LCCs. Drug resistance is an important malignant phenotype of CSCs. The IC50 values of MYH9 knockdown cells and control cells to paclitaxel were $5.599 \mathrm{nM}$ and $15.12 \mathrm{nM}$ (Fig. $2 \mathrm{H}$ ), respectively, indicating that the sensitivity of LCCs to paclitaxel increased with the decrease of MYH9.

Tumorigenicity is one of the most important characteristics of CSCs. To observe the effect of MYH9 on the growth of LCCs in vivo, cells stably knocked down by MYH9 grew slowly in nude mice, and the volume and weight of xenografts were significantly smaller than those in the control group (Fig. 2l). Also, IHC was performed to verify the role of MYH9 in the growth, invasion, and stemness of lung cancer xenografts. The expression of MYH9 in knockout xenografts was lower than that in the control group, and the expression of Ki67 was also significantly downregulated.

In contrast, the results of $\mathrm{N}$-cadherin, CD44, and CD133 were consistent with Western blot. After the knockout of MYH9, the expression in xenografts decreased (Fig. 2J). These results showed that the malignant phenotype of tumors decreased after the down-regulated expression of MYH9, indicating that MYH9 affects the development of lung cancer by regulating LCSC characteristics.

\section{Overexpression of MYH9 enhances the stemness characteristics of LCCs in vivo and in vitro}

A549 cell line with low endogenous expression of MYH9 has constructed a stable cell line with overexpression (Fig. 3A). Compared with the control cells, colony formation and proliferation were significantly enhanced after overexpression of MYH9 (Fig. 3B, C). Also, the self-renewal ability of MYH9 overexpression cells was significantly enhanced (Fig. 3D), and the expression levels of CD44, SOX2, Nanog, CD133, and OCT4 were significantly upregulated (Fig. 3E).

The migration and invasion ability of A549-MYH9 cells was stronger than that of A549-Vector cells (Fig. 3F), and N-cadherin, Vimentin, and Snail increased in A549-MYH9 cells (Fig. 3G). Paclitaxel resistance test showed that overexpression of MYH9 enhanced the drug resistance of LCCs (Fig. $3 \mathrm{H}$ ). These results suggested that $\mathrm{MYH} 9$ can regulate the stemness characteristics of LCCs, and subsequent experiment in vivo showed that A549MYH9 cells formed xenografts faster than A549-Vector cells, with larger volume and weight (Fig. 3I). In the xenografts with high expression of MYH9, the expression of Ki67, N-cadherin, CD44 and CD133 were upregulated (Fig. 3J).

\section{Blebbistatin reduced the tumorigenicity of $\mathrm{MYH9}$ in vivo}

Blebbistatin is currently recognized as an inhibitor of MYH9. To further prove the effectiveness of $\mathrm{MYH} 9$, results revealed that the blebbistatin could significantly inhibit transplanted tumor growth and volume (Fig. 4A, B).

Simultaneously, the inhibition rates of low, middle, and high doses $(10 \mathrm{mg} / \mathrm{kg}, 20 \mathrm{mg} / \mathrm{kg}$, and $40 \mathrm{mg} / \mathrm{kg})$ of blebbistatin on the tumor weight of the xenografts were $23.4 \%, 40.2 \%$, and $83.4 \%$, respectively, with a significant difference and dose-dependent effect (Fig. 4C). IHC analysis showed that the expression of MYH9 and $\mathrm{Ki} 67$ in the low, middle, and high dose $(10 \mathrm{mg} / \mathrm{kg}, 20 \mathrm{mg} / \mathrm{kg}$, and $40 \mathrm{mg} / \mathrm{kg}$ ) groups of blebbistatin decreased gradually and correlated with the concentration of inhibitor (Fig. 4D). These results suggested that the blebbistatin could inhibit tumor growth in nude mice, which further proved that MYH9 can regulate the stem characteristics of LCCs.

\section{MYH9 regulates stem phenotype of LCCs by activating the mTOR signaling pathway}

To explore the specific mechanism of the effect of MYH9 on the stem phenotype of LCCs, the total proteins extracted from MYH9 knockdown and overexpression cells were used to detect the stemness-related signal pathway [32-34]. The results showed that MYH9 could activate different branch pathways of the mTOR pathway. In the LRP6/GSK3/mTOR signaling pathway, phosphorylated LRP6 in MYH9 overexpressed LCCs affected phosphorylation of GSK-3a $a^{\text {Ser21 }}$ and GSK-3 $\beta^{\text {Ser9 }}$ by upregulating DVL2. It then phosphorylated mTOR-negative regulator TSC2 and finally increased the level of mTOR phosphorylation. After MYH9 knockdown, the expression of these proteins showed the opposite trend (Fig. 5A); After MYH9 overexpression, the PI3K/AKT/mTOR signaling pathway was activated, whereas after MYH9 knockdown, the pathway was inhibited (Fig. 5B). This result indicated that MYH9 regulates the stemness of LCCs by activating the mTOR signaling pathway.

To further identify whether MYH9 acts through the mTOR signal pathway, the mTOR inhibitor Rapamycin and GSK3 inhibitor (CHIR-99021) were used to detect the self-renewal ability of LCCs. The results showed that MYH9 significantly promoted the self-renewal ability of A549 cells, while the addition of inhibitor Rapamycin blocked the effect of MYH9 on the self-renewal ability (Fig. 5C). Simultaneously, the result of MYH9 knockdown cells treated with CHIR-99021 showed that the self-renewal ability of MYH9 knockdown cells increased after the addition of inhibitor CHIR-99021 (Fig. 5D). These results suggest that MYH9 regulates the malignant stem phenotype of LCCs through the mTOR signal pathway.

MYH9 is an independent risk factor in patients with NSCLC To study the clinical significance of MYH9, the expression of MYH9 in NSCLC tissue microarrays by IHC is detected. The clinicopathological features of patients with NSCLC are given in Additional file 1 , schedule 1. The $\mathrm{IHC}$ results showed that the expression of $\mathrm{MYH} 9$ in NSCLC was stronger than that in matched paracancerous tissues (Fig. 6A). Also, according to the expression intensity of MYH9, patients with NSCLC were divided into four grades: negative $(-)$, weakly positive $(+)$, positive $(++)$ and strongly positive $(+++)$ (Fig. 6B). Furthermore, patients with NSCLC were divided into the MYH $9^{\text {high }}\left(++\right.$ and +++ are high) and $\mathrm{MYH} 9^{\text {low }}$ $(-$ and + are low).The results showed that there was a significant 
A

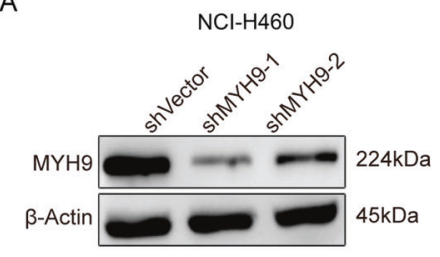

C
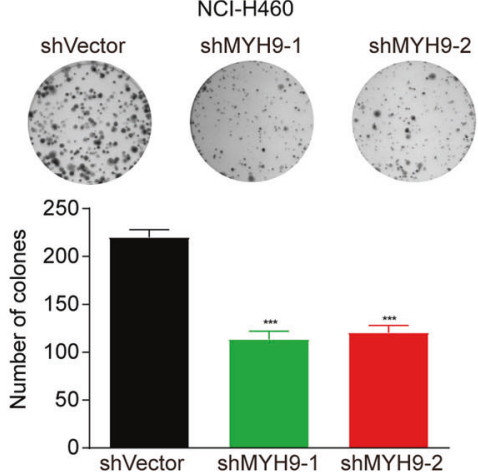

E

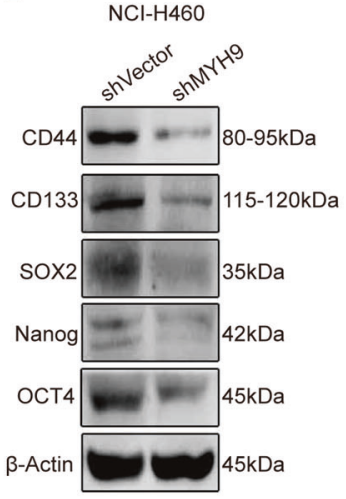

$\mathrm{H}$

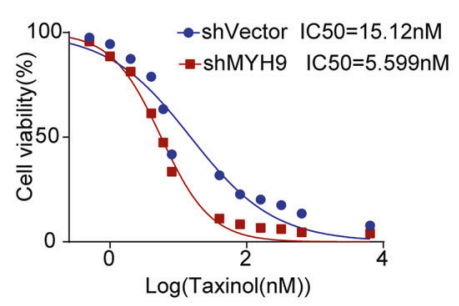

G

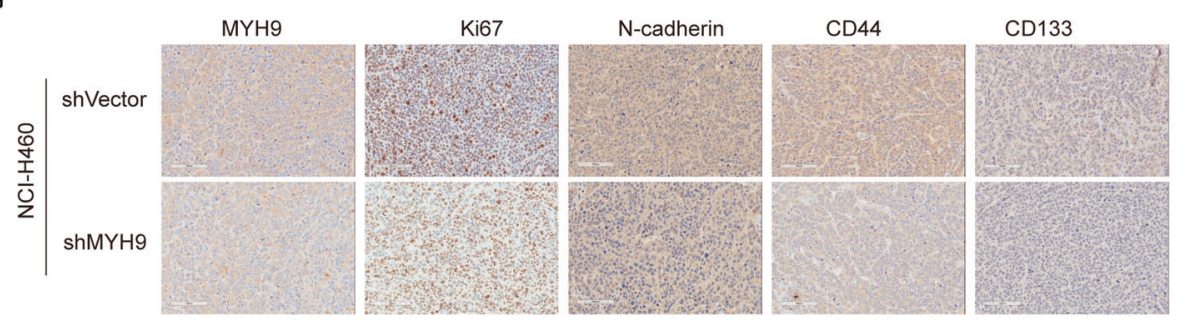

B
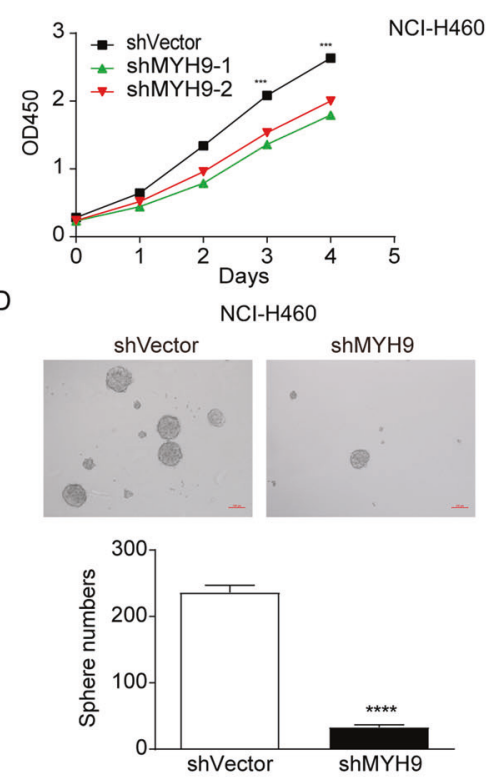

G

I

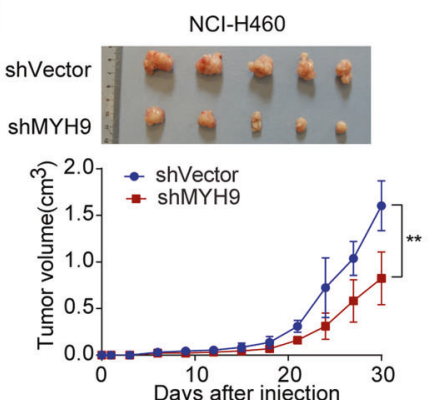

F

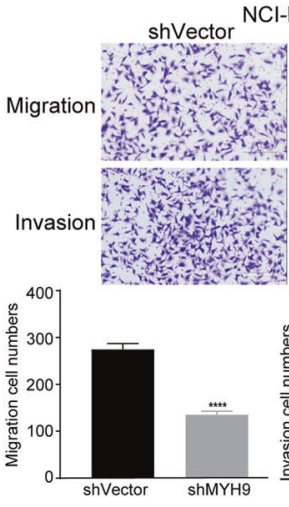

ShMYH9
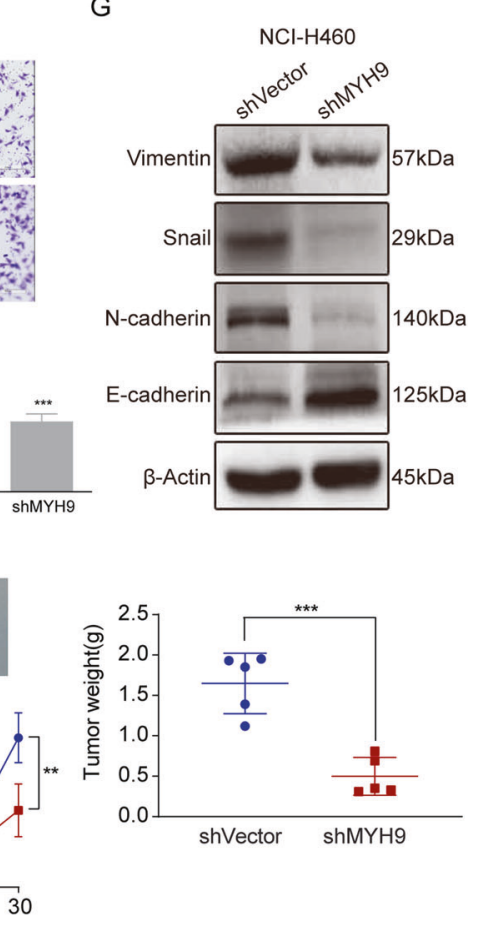

Fig. 2 Downregulation of MYH9 expression suppresses the stem cell-like malignant phenotype of LCCs in vivo and in vitro. A The protein expression of MYH9 in NCl-H460-shMYH9 stable transformant and control cells. B, C Proliferation ability of NCl-H460-shMYH9 and NCl-H460shVector cells (mean $\pm \mathrm{SD} ; n=3 ;{ }^{* * *} p<0.001$; Student's $t$-test). D Test results of self-renewal ability of NCI-H460-shMYH9 and NCl-H460-shVector cells (mean $\pm \mathrm{SD} ; n=3$; ${ }^{* * *} p<0.0001$; Student's $t$-test). E The expression of CD44, SOX2, CD133, OCT4, and Nanog in NCI-H460-shMYH9 and NCI-H460shVector cells. F Analysis of migration and invasion ability of MYH9 stable knockdown and control cells (scale bar, $200 \mu \mathrm{m} ;$ mean \pm SD; $n=3 ; * * * p<$ 0.001 and ${ }^{* * * *} p<0.0001$; Student's $t$-test). G Western blot of EMT marker in NCl-H460-shMYH9 and NCl-H460-shVector cells. H Analysis of drug resistance of $\mathrm{NCl}-\mathrm{H} 460-$-shMYH9 and $\mathrm{NCl}-\mathrm{H} 460-$-shVector cells to paclitaxel. I Analysis of the effect of MYH9 knockout on the growth of LCCs in vivo by subcutaneous tumor formation assay (mean \pm SD; $n=5$; ** $p<0.01$ and ${ }^{* * *} p<0.001$; Student's $t$-test). J IHC analysis of N-cadherin, MYH9, CD133, CD44, and Ki67 in xenografts of NCl-H460-shMYH9 and NCl-H460-shVector cells (scale bar, $100 \mu \mathrm{m} ; n=5$ ). 
A

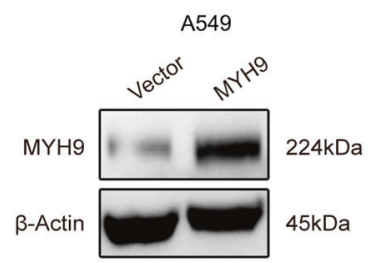

C
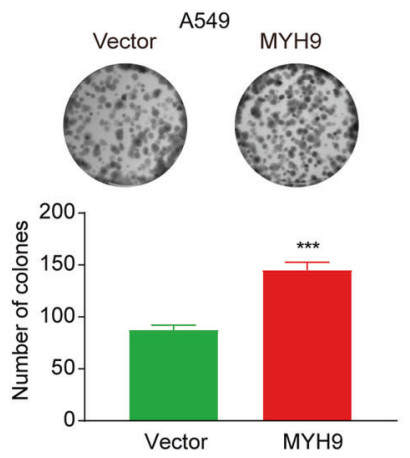

B

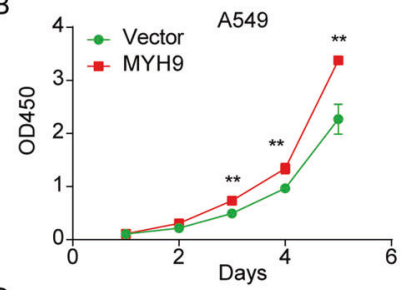

D
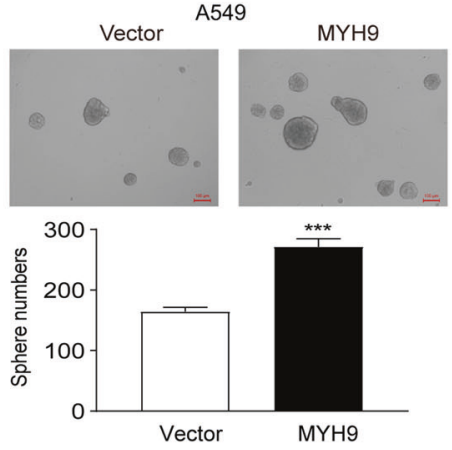

E

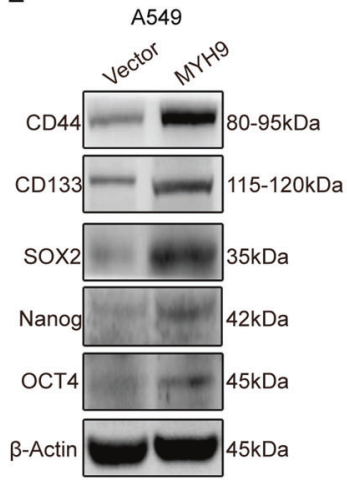

$\mathrm{H}$

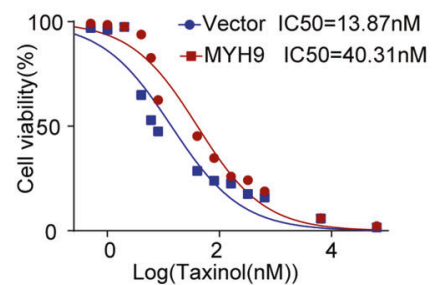

G

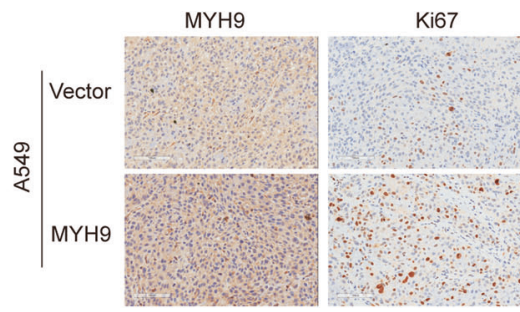

F

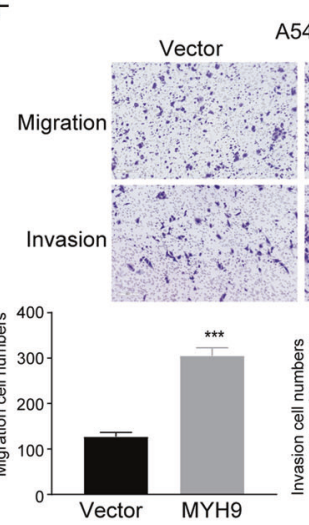

A549

MYH9<smiles>[131In]</smiles>

I

G
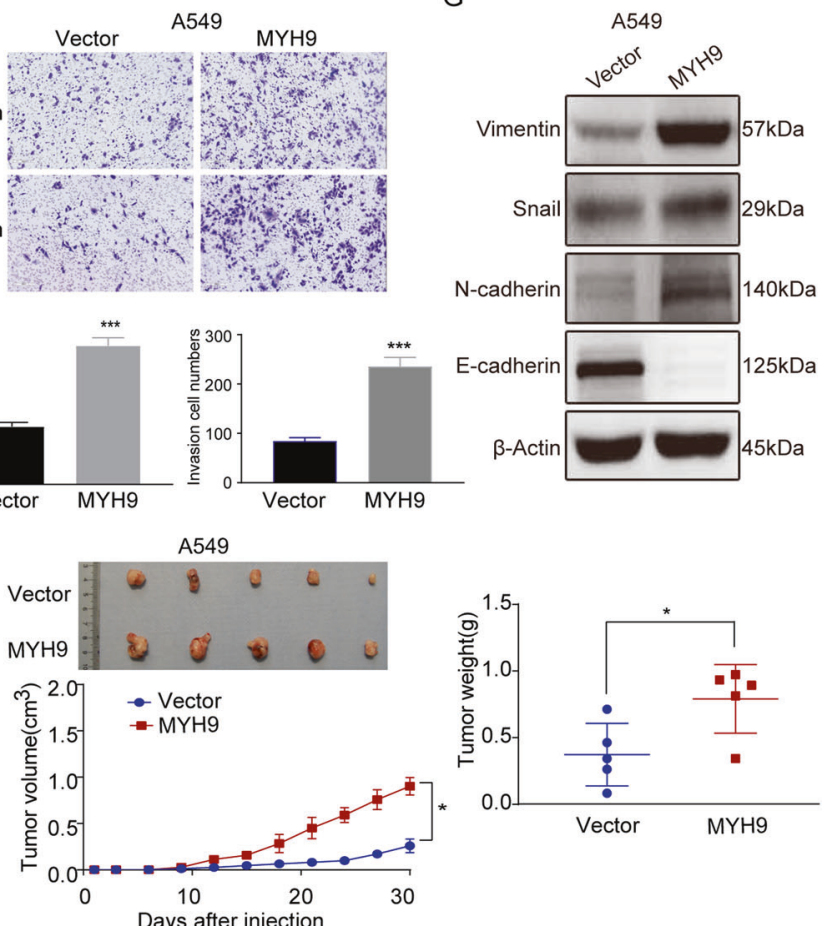

Fig. 3 Overexpression of MYH9 enhances the stemness characteristics of LCCs in vivo and in vitro. A The protein expression of MYH9 in A549-MYH9 and A549-Vector cells. B, C Proliferation ability of A549-MYH9 and A549-Vector cells (mean \pm SD; $n=3 ;{ }^{* *} p<0.01$ and ${ }^{* * *} p<0.001$; Student's $t$-test). D Self-renewal ability of A549-MYH9 and A549-Vector cells (mean \pm SD; $n=3$; ${ }^{* * *} p<0.001$; Student's $t$-test). E The expression levels of CD44, SOX2, CD133, OCT4, and Nanog in MYH9 overexpressed and control cells. F Migration and invasion ability of A549-MYH9 and A549-Vector cells (scale bar, $200 \mu \mathrm{m} ;$ mean \pm SD; $n=3$; ${ }^{* * *} p<0.001$; Student's $t$-test). G Expression of E-cadherin, Snail, Vimentin, and N-cadherinin A549-MYH9 and A549-Vector cells. $\mathbf{H}$ Analysis of drug resistance of MYH9-overexpressing and control cells to paclitaxel. I Growth of xenografts of A549-MYH9 and A549-Vector cells in nude mice (mean \pm SD; $n=5 ;{ }^{*} p<0.05$ and ${ }^{* *} p<0.01$; Student's $t$-test). J Analysis of the expression of MYH9, Ki67, Ncadherin, CD133, and CD44in transplanted tumor tissues of A549-MYH9 and A549-Vector cells (scale bar, $100 \mu \mathrm{m} ; n=5$ ). 
A

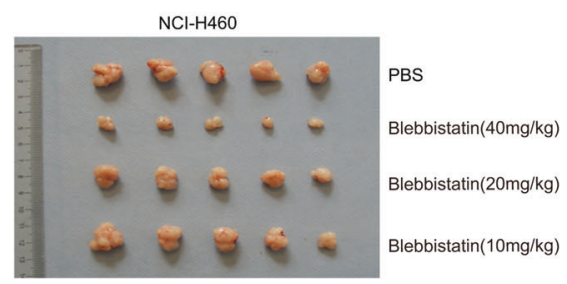

C

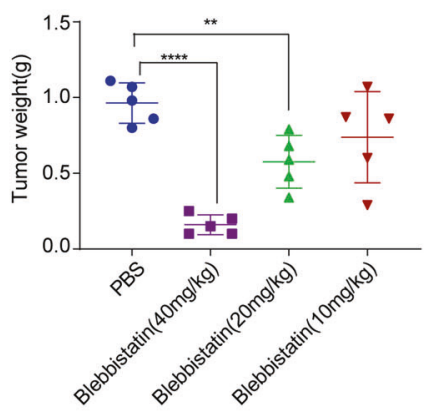

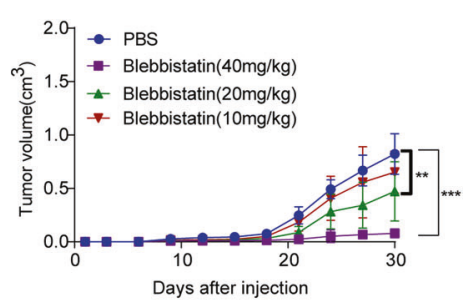

$\mathrm{D}$

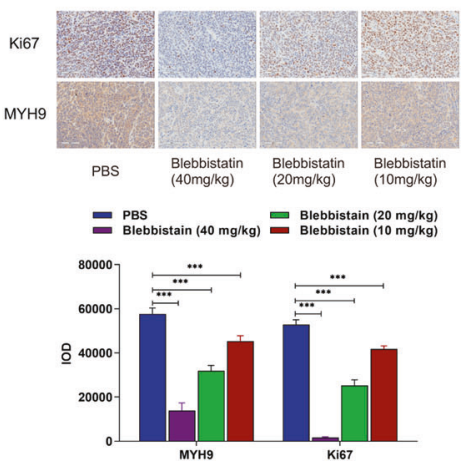

Fig. 4 Blebbistatin reduced tumorigenicity of MYH9 in vivo. A Xenografts were formed in the PBS group and high, middle, and low dose $(40 \mathrm{mg} / \mathrm{kg}, 20 \mathrm{mg} / \mathrm{kg}$, and $10 \mathrm{mg} / \mathrm{kg})$ groups of blebbistatin. B Growth curve of xenografts in different dose groups of blebbistatin and PBS group $\left(n=5 ;{ }^{* *} p<0.01\right.$ and ${ }^{* * *} p<0.001$; Student's $t$-test). C Tumor weights of xenografts in high, middle, and low dose groups of blebbistatin and PBS group ( $n=5 ;{ }^{* *} p<0.01$ and ${ }^{* * * *} p<0.0001$; Student's $t$-test). D Expression of MYH9 and Ki67 in xenograft tissues of different dose groups of blebbistatin and PBS groups (scale bar, $100 \mu \mathrm{m} ; n=5$ ).

correlation between the expression of MYH9 and the survival and prognosis of patients $(p=0.0011)$. The overall survival time of patients with up-regulated MYH9 expression (43 months) was significantly shorter than that of patients with low expression of MYH9 (72 months) (Fig. 6C), indicating that MYH9 can predict the survival time of patients with NSCLC.

MYH9 expression in the cell membrane, cytoplasm, and nucleus and survival prognosis of patients with lung cancer were 0.0066 , 0.0350 and 0.0368 (Fig. 6D-F), respectively, which further confirmed that MYH9 was closely related to the prognosis of patients with NSCLC. It has the significance of predicting the survival and prognosis of patients. Simultaneously, univariate regression analysis showed that in addition to the expression of MYH9, the patient's age, lymph node metastasis, and clinical stage were also related to the overall survival time. Multivariate Cox regression analysis showed that age, sex, clinical stage, and MYH9 expression were independent risk factors for the overall survival of patients with NSCLC, which could independently evaluate patient survival prognosis with NSCLC (Table 1).

\section{DISCUSSION}

The high morbidity, high mortality, and low survival rate of patients with NSCLC are closely related to CSCs, which have the biological characteristics of unlimited self-renewal, high tumorigenicity, high invasion, and migration, and strong drug resistance [35-38]. The study found that MYH9 can regulate the stemnessrelated malignant characteristics of LCCs and is related to the overall survival of patients with NSCLC.MYH9, as a heavy chain of nonmuscle myosin $\mathrm{II}$, is a microfilament cytoskeleton-related protein, which has various functions, such as participating in cell adhesion, migration, invasion, and regulating cell signal transduction and material transport. Through clinical tissue samples of gastric cancer, MYH9 was positively correlated with the depth of invasion and lymph node metastasis of gastric cancer, and MYH9 promoted the progression of esophageal cancer, which was closely related to lymph node metastasis and pathological grade of patients with esophageal cancer. The expression of MYH9 is upregulated in other cancers, such as colorectal cancer, head and neck cancer, and acute myeloid leukemia, and is associated with poor prognosis of these cancers [26, 27, 39-41]. Also, Arora et al. found that MYH9 can regulate the biological behavior of stem cells $[30,31]$. These studies have suggested that MYH9 affects tumor progression by regulating the phenotype of CSCs.

In this study, MYH9 was enrichment expression in many kinds of LCCs from serum-free medium by flow cytometry, indicating that MYH9 can identify LCSCs and is related to the stem phenotype of tumors. Subsequently, we constructed a stable overexpression and knockdown cell line of MYH9 and studied the effect of MYH9 on stem characteristics from LCCs in vivo and in vitro. These results revealed that the ability of drug resistance, self-renewal, migration and invasion and proliferation of LCCs decreased significantly after the down-regulated expression of MYH9, and the expression levels of $\mathrm{N}$-cadherin, Vimentin, E-cadherin and Snail and stem cell markers OCT4, CD133, CD44, SOX2 and Nanog also showed a downward trend. Furthermore, tumor formation tests in vivo showed that the xenografts formed by LCCs knocked down by MYH9 grew slowly, tumor size was smaller, and tumor weight was lighter, and the expression trend of metastasis molecule $\mathrm{N}$ cadherin, malignant proliferation marker Ki67 and stem cell markers CD44 and CD133 in xenografts was the same as that of MYH9. In contrast, overexpression of MYH9 reversed the noted phenotypic changes. These studies suggested that MYH9 can regulate the stem phenotype of LCCs, which may be related to tumor formation and development. Subsequently, we used blebbistatin, a specific inhibitor of MYH9, to treat lung cancer in vivo, and found that after treatment with blebbistatin, the growth of LCCs was inhibited in a dose-dependent manner

To explore the molecular mechanism, we found that MYH9 plays its role by activating different branches of the mTOR signaling pathway: on the one hand, phosphorylated LRP6 affects the phosphorylation of GSK-3 by regulating DVL2 and then 
A

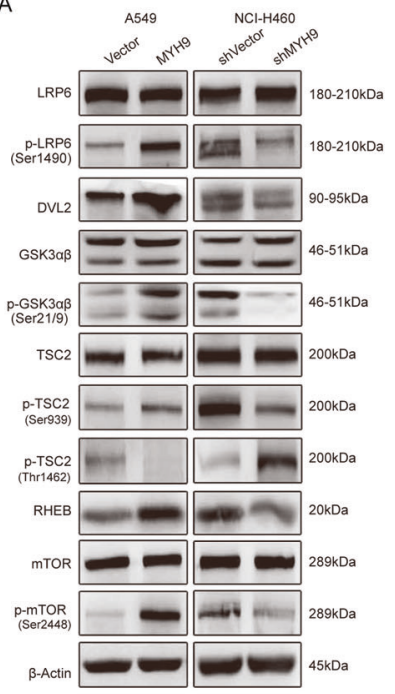

B

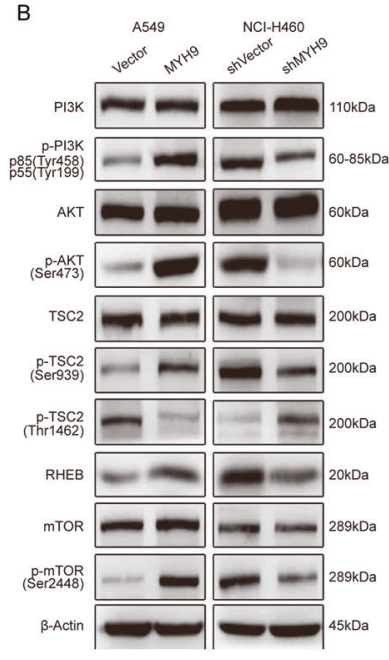

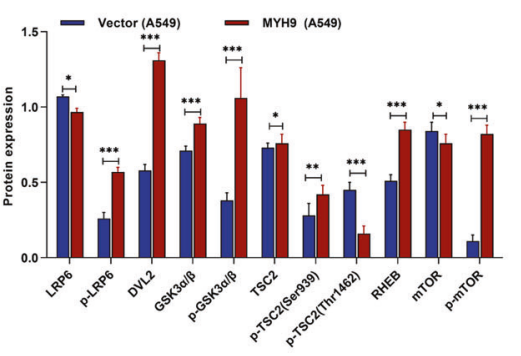

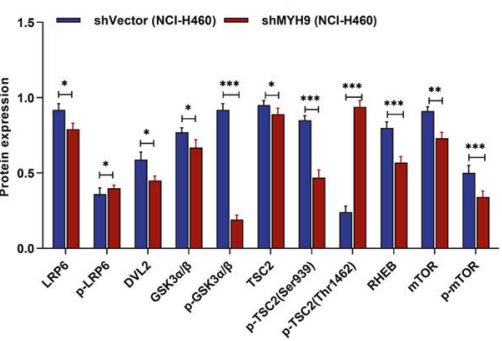

C

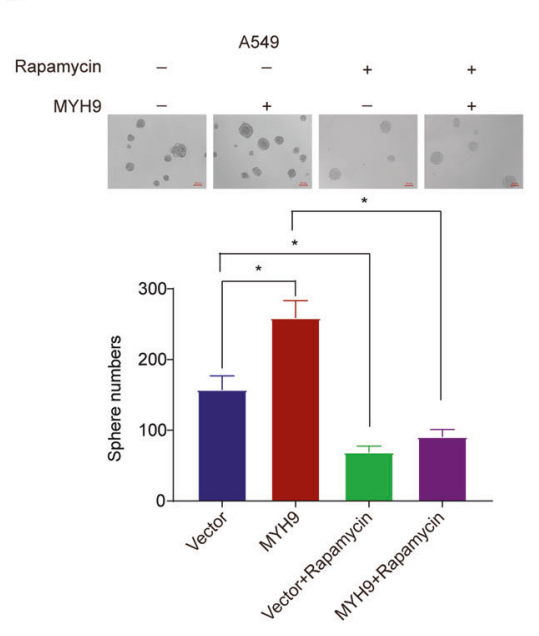

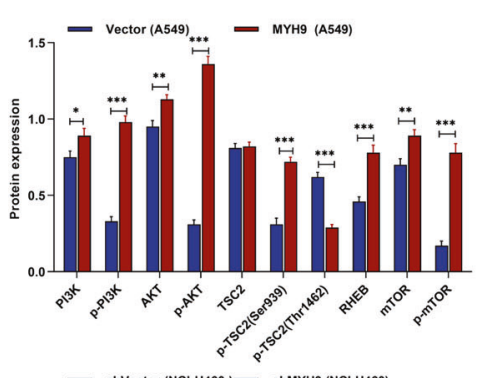

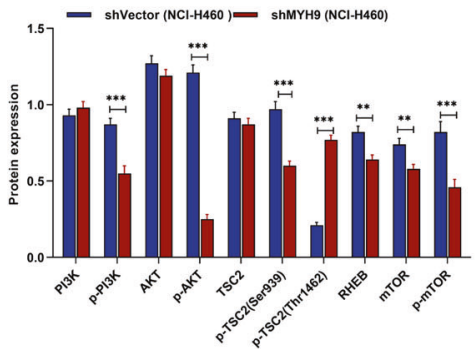

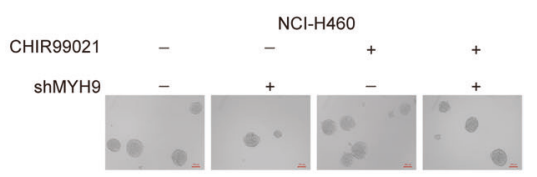

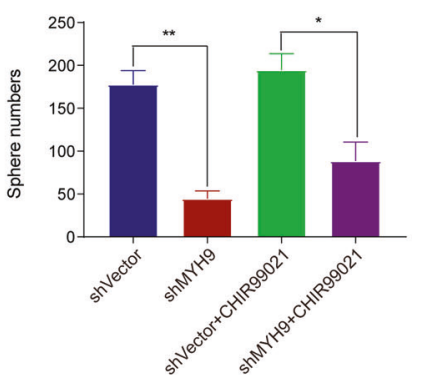

Fig. 5 MYH9 regulates the stem phenotype of LCCs by activating the mTOR signaling pathway. A The protein expression of LRP6/GSK3/ mTOR signal pathway markers in MYH9 stable transformant and control cells. B The expression of proteins from PI3K/AKT/mTOR signaling pathway in MYH9 stable transgenic and control cells. C Analysis of the effect of mTOR inhibitor Rapamycin on the self-renewal ability of MYH9 overexpressing cells and control cells (scale bar, $100 \mu \mathrm{m} ; n=3 ;{ }^{*} p<0.05$; n.s., not significant; Student's $t$-test). D The effect of GSK3 inhibitor CHIR-99021 on MYH9 low expression cell self-renewal ability and control cells (scale bar, $100 \mu \mathrm{m} ; n=3$; ${ }^{*} p<0.05$ and ${ }^{* *} p<0.01$; Student's $t$-test).

regulates the negative regulator of mTOR to change the level of mTOR phosphorylation; on the other hand, the expression of MYH9 affects the activation of PI3K/AKT/mTOR signaling pathway. Then, the mTOR inhibitor Rapamycin and GSK3a/ $\beta$ inhibitor CHIR99021 was used to verify this molecular mechanism. It demonstrated that MYH9 regulates the stem phenotype of LCCs through the mTOR signal pathway.

Finally, we found that MYH9 was highly expressed in clinical samples of NSCLC, and expression in the cell membrane and nucleus was specific to that of samples. Although some studies have shown that the expression of MYH9 is related to the histological characteristics of lung adenocarcinoma [29], more evidence is needed to prove its therapeutic value in patients with NSCLC. Univariate and multivariate analysis showed that MYH9 was positively correlated with short overall survival and poor prognosis and was an independent risk factor for patients with NSCLC.
In conclusion, our research showed that MYH9 is a potential marker of LCSC, which was enriched in spheroid cells, which affected malignant stem phenotypes of LCCs by regulating the mTOR signal pathway. The expression of MYH9 was positively correlated with the survival and prognosis of patients with lung cancer and can be used as an independent index, providing a new potential target for treating patients with lung cancer.

\section{MATERIALS AND METHODS}

\section{Tissue microarrays}

The tissue microarrays were purchased from Shanghai Xinchao Biotechnology (China). The pathological data of clinical specimens are summarized in Tables S1. The study was approved by the medical ethics committee of Cancer Hospital, Chinese Academy of Medical Sciences (Beijing, China) (Ethical approval number: NCC1999 G-003). 
A

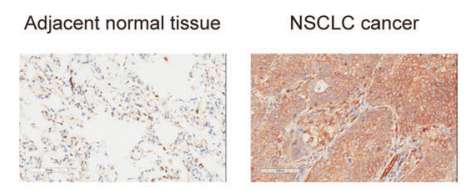

C

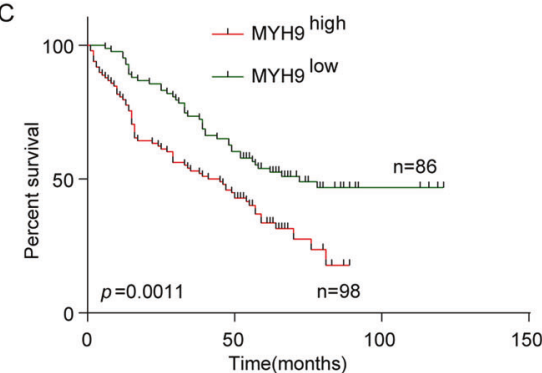

$\mathrm{E}$

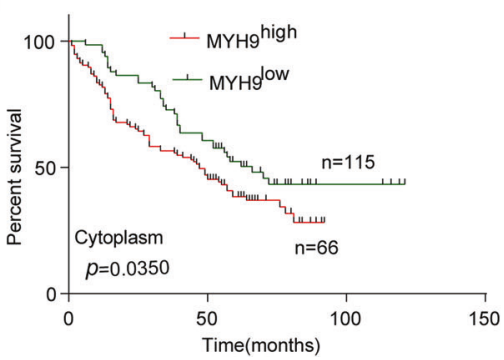

B
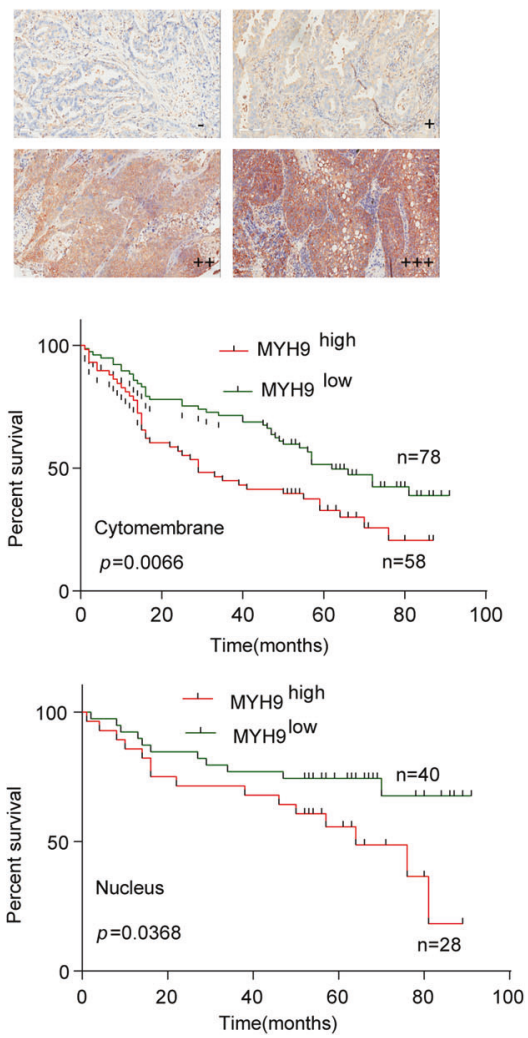

Fig. 6 MYH9 is an independent risk factor in patients with NSCLC. A Expression of MYH9 in NSCLC and paracancerous tissues (scale bar, $100 \mu \mathrm{m})$. B IHC divided the expression of MYH9 in lung cancer into four grades: negative (-), weakly positive $(+)$, positive $(++)$, and strongly positive $(+++)($ scale bar, $100 \mu \mathrm{m})$. C Kaplan-Meier survival curve of overall survival in patients with NSCLC $\left({ }^{* *} p<0.01 ;\right.$ Log-rank test). D, E Kaplan-Meier survival curve and univariate analysis were used to study the correlation between MYH9 expression in the cell membrane, cytoplasm, nucleus, and survival prognosis in patients with NSCLC $\left({ }^{*} p<0.05\right.$ and ${ }^{* *} p<0.01$; Log-rank test).

Table 1. Univariate and multivariate regression analysis of prognosis in patients with NSCLC.

\begin{tabular}{|c|c|c|c|c|}
\hline \multirow[t]{2}{*}{ Features } & \multicolumn{2}{|c|}{ univariate analysis } & \multicolumn{2}{|c|}{ multivariable analysis } \\
\hline & $\mathrm{HR}(95 \% \mathrm{Cl})$ & $p$ value & $\operatorname{HR}(95 \% \mathrm{Cl})$ & $p$ value \\
\hline Age(years), $\geq 65 v<65$ & $1.55(1.06-2.27)$ & 0.023 & $1.73(1.16-2.56)$ & 0.007 \\
\hline Tumor Volume, $>18 \mathrm{v} \leq 18$ & $1.04(0.70-1.53)$ & 0.862 & & \\
\hline LNM, Positive v Negative & $2.40(1.61-3.58)$ & 0.000 & & \\
\hline Tumor differentiation & $1.05(0.70-1.59)$ & 0.816 & & \\
\hline AJCC stage & $2.27(1.53-3.37)$ & 0.000 & $1.78(0.95-3.30)$ & 0.009 \\
\hline
\end{tabular}

Note: $L N M$ lymph node metastasis, $H R$ hazard ratio, $95 \% \mathrm{Cl}=95 \%$ confidence interval.

\section{Cell culture}

Cell lines A549, NCl-H1299, GLC-82, and NCl-H460 were preserved at the Laboratory of Cell and Molecular Biology, Institute of Oncology, Chinese Academy of Medical Sciences. SPCA-1 and NCI-H226 cell lines were purchased from the Shanghai Cell Bank of the Chinese Academy of Sciences. After adding fetal bovine serum (FBS) to the RPMI-1640 medium, the cells were cultured at $37^{\circ} \mathrm{C}$ in $5 \% \mathrm{CO}_{2}$. All of the cell lines were confirmed to be free of mycoplasma contamination after testing with the kit from Shanghai Yise Medical Technology (MD001).

\section{Self-renewal assay}

The LCCs were inoculated into a low adhesion plate (Corning) with laying 500 cells in each well and cultured in a serum-free DMEM/F12 medium containing $0.8 \%$ methylcellulose added with $20 \mathrm{ng} / \mathrm{ml} \mathrm{EGF,} 10 \mathrm{ng} / \mathrm{mL} \mathrm{LIF}$,
$20 \mathrm{ng} / \mathrm{mL} \mathrm{bFGF}$, and B27 (1:50) factors. The fresh medium was added every three days. After 14 days, the formed spheres were photographed and counted.

\section{Antibodies for western blot, immunofluorescence and Immunohistochemistry}

MYH9 (\#M8064; Sigma), CD133 (\#ab19898; Abcam), Nanog (\#3580; CST), OCT4 (\#2750; CST), CD44 (\#3570; CST), SOX2 (\#3579; CST), Vimentin (\#3932; CST), Snail (\#3879; CST), N-Cadherin (\#13116; CST), E-Cadherin (\#20874-1AP; Proteintech), LRP6 (\#2560; CST), Phospho-LRP6Ser1490(\#2568; CST), DVL2 (\#12037-1-AP; Proteintech), GSK-3a/b (\#5676; CST), Phospho-GSK-3a/ bSer21/9 (\#9331; CST), Phospho-TSC2 Antibody Sampler Kit (\#8350; CST), RHEB (\#15924-1-AP; Proteintech), mTOR (\#2983; CST), Phospho-mTORSer2448(\#5536; CST), and b-Actin (\#4970; CST). 


\section{Flow cytometry}

The LCCs were digested, centrifuged, and incubated with antibody MYH9 (\#M8064; Sigma) at $25^{\circ} \mathrm{C}$ for $1 \mathrm{~h}$. Then incubated with fluorescent antibody 488 (\#711-486-152; Jackson ImmunoResearch;) for $0.5 \mathrm{~h}$. PBS was rinsed and detected by AccuriC6cytometer (BD Biosciences).

\section{Proliferation, colony formation, and drug resistance assay} After the LCCs were digested, $3.5 \times 10^{3}$ cells were cultured for 5 days, the reaction time was $2.5 \mathrm{~h}$ after adding $20 \mu \mathrm{L}$ of CCK-8 assay kit (Dojindo). Finally, the absorbance at $450 \mathrm{~nm}$ wavelength was determined by an enzyme-labeling instrument (Bio-rad). $1 \times 10^{3}$ cells were inoculated and cultured for 14 days, then fixed, stained and dried. Finally, the clones were photographed using a microscope, and the clone formation rate was calculated. $4 \times 10^{3}$ cells were inoculated and cultured for $24 \mathrm{~h}$. Then the cells were cultured in the medium containing the concentration gradient of paclitaxe. After $72 \mathrm{~h}$, add CCK-8 assay kit solution and reacted for $2.5 \mathrm{~h}$. The OD450 was detected by an enzyme-labeling instrument

\section{Migration and invasion analysis}

The medium without FBS and Matrigel (BD Biosciences) were added to the Transwell chamber (8- $\mu \mathrm{m}$ pore size, Millipore). The $3 \times 10^{4}$ cells were inoculated with serum-free medium in the upper chamber of the plate. Finally, five visual fields for each well were counted under the microscope. In the migration assay, Matrigel was not added in the chamber, the other steps were the same as in the invasion assay.

\section{Tumorigenicity and treatment in nude mice}

Female BALB/c nude mice (4-5 weeks old) were purchased from Huafukang Company. For tumorigenesis test, $3 \times 10^{6}$ cells were injected into each nude mice. The animals were killed when the tumor's length and diameter were $1-2 \mathrm{~cm}$, and the tumor was photographed and weighed.

For blebbistatin treatment test, $3 \times 10^{6}$ cells were inoculated under the axilla of the forelimbs of mice. Nude mice were divided into four groups: PBS group, high-dose group ( $40 \mathrm{mg} / \mathrm{kg}$ ), medium-dose group ( $20 \mathrm{mg} / \mathrm{kg})$, and lowdose group $(10 \mathrm{mg} / \mathrm{kg}) ; 0.4 \mathrm{~mL}$ blebbistatin was injected intraperitoneally in each nude mice. The nude mice were treated once every other day for four weeks. Other steps were the same as tumorigenesis assay.

\section{Statistical analysis}

Analysis of variance (ANOVA) or Student's $t$-test was used to compare the results between experimental data groups, and the value was expressed as means \pm SD. Spearman analysis compared the correlation between the two; Fisher exact test and chi-square test compared the correlation between clinical parameters; Cox proportional hazard regression model Log-rank test and Kaplan-Meier were used for survival analysis statistical. GraphPad Prism7.0 and SPSS software were used for data analysis, and the statistically significant standard value was $p<0.05$.

\section{DATA AVAILABILITY}

The datasets generated during and/or analysed during the current study are available from the corresponding author on reasonable request.

\section{REFERENCES}

1. Torre LA, Bray F, Siegel RL, Ferlay J, Lortet-Tieulent J, Jemal A. Global cancer statistics, 2012. CA Cancer J. Clin. 2015;65:87-108.

2. Torre LA, Siegel RL, Ward EM, Jemal A. Global cancer incidence and mortality rates and trends-an update. Cancer Epidemiol. Biomark. Prev. 2016;25:16-27.

3. Akbari-Birgani S, Paranjothy T, Zuse A, Janikowski T, Cieslar-Pobuda A, Likus W, et al. Cancer stem cells, cancer-initiating cells and methods for their detection. Drug Disco Today. 2016;21:836-42.

4. Cooper J, Giancotti FG. Integrin signaling in cancer: mechanotransduction, stemness, epithelial plasticity, and therapeutic resistance. Cancer Cell. 2019;35:347-67.

5. Dawood S, Austin L, Cristofanilli M. Cancer stem cells: implications for cancer therapy. Oncology 2014;28:1101-1107.

6. Wang T, Shigdar S, Gantier MP, Hou Y, Wang L, Li Y, et al. Cancer stem cell targeted therapy: progress amid controversies. Oncotarget. 2015;6:44191-206.

7. Kim CF, Jackson EL, Woolfenden AE, Lawrence S, Babar I, Vogel S, et al. Identification of bronchioalveolar stem cells in normal lung and lung cancer. Cell. 2005;121:823-35
8. Ho MM, Ng AV, Lam S, Hung JY. Side population in human lung cancer cell lines and tumors is enriched with stem-like cancer cells. Cancer Res. 2007:67:4827-33.

9. Eramo A, Lotti F, Sette G, Pilozzi E, Biffoni M, Di Virgilio A, et al. Identification and expansion of the tumorigenic lung cancer stem cell population. Cell Death Differ. 2008;15:504-14.

10. Jiang F, Qiu Q, Khanna A, Todd NW, Deepak J, Xing L, et al. Aldehyde dehydrogenase 1 is a tumor stem cell-associated marker in lung cancer. Mol. Cancer Res. 2009;7:330-338.

11. Liang $D$, Shi Y. Aldehyde dehydrogenase-1 is a specific marker for stem cells in human lung adenocarcinoma. Med Oncol. 2012;29:633-639.

12. Nishino M, Ozaki M, Hegab AE, Hamamoto J, Kagawa S, Arai D, et al. Variant CD44 expression is enriching for a cell population with cancer stem cell-like characteristics in human lung adenocarcinoma. J. Cancer. 2017;8:1774-85.

13. Zhao $C$, Chen $A$, Jamieson $C H$, Fereshteh $M$, Abrahamsson A, Blum J, et al. Hedgehog signalling is essential for maintenance of cancer stem cells in myeloid leukaemia. Nature. 2009;458:776-779.

14. Alamgeer M, Peacock CD, Matsui W, Ganju V, Watkins DN. Cancer stem cells in lung cancer: evidence and controversies. Respirology. 2013;18:757-64.

15. Richter S, Bedard PL, Chen EX, Clarke BA, Tran B, Hotte SJ, et al. A phase I study of the oral gamma secretase inhibitor R04929097 in combination with gemcitabine in patients with advanced solid tumors (PHL-078/CTEP 8575). Invest N. Drugs. 2014;32:243-249.

16. Yang T, Rycaj K. Targeted therapy against cancer stem cells. Oncol. Lett. 2015; 10:27-33.

17. Pecci A, Ma X, Savoia A, Adelstein RS. MYH9: Structure, functions and role of nonmuscle myosin IIA in human disease. Gene. 2018;664:152-67.

18. Billington N, Wang A, Mao J, Adelstein RS, Sellers JR. Characterization of three fulllength human nonmuscle myosin II paralogs. J. Biol. Chem. 2013;288:33398-410.

19. Ma X, Adelstein RS. The role of vertebrate nonmuscle Myosin II in development and human disease. Bioarchitecture. 2014;4:88-102.

20. Galeano D, Zanoli L, L'Imperio V, Fatuzzo P, Granata A. [Renal diseases related to MYH9 disorders]. G Ital. Nefrol. 2017;34:40-57.

21. Girolami A, Sambado L, Bonamigo E, Vettore S, Lombardi AM. Occurrence of thrombosis in congenital thrombocytopenic disorders: a critical annotation of the literature. Blood Coagul. Fibrinolysis. 2013;24:18-22.

22. Okano S, Takase M, Iseki K, Toriumi N, Kaneda M, Kunishima S. Genotypephenotype Correlation of the p.R1165C Mutation in the MYH9 Disorder: Report of a Japanese Pedigree. J. Pediatr. Hematol. Oncol. 2015;37:e352-355.

23. Smith AS, Pal K, Nowak RB, Demenko A, Zaninetti C, Da Costa L, et al. MYH9-related disease mutations cause abnormal red blood cell morphology through increased myosin-actin binding at the membrane. Am J Hematol. 2019;94:667-677.

24. Derycke L, Stove C, Vercoutter-Edouart AS, De Wever O, Dolle L, Colpaert N, et al. The role of non-muscle myosin IIA in aggregation and invasion of human MCF-7 breast cancer cells. Int J Dev Biol. 2011:55:835-40.

25. Liu T, Ye Y, Zhang X, Zhu A, Yang Z, Fu Y, et al. Downregulation of nonmuscle myosin IIA expression inhibits migration and invasion of gastric cancer cells via the cJun Nterminal kinase signaling pathway. Mol. Med Rep. 2016;13:1639-44.

26. Wang B, Qi X, Liu J, Zhou R, Lin C, Shangguan J, et al. MYH9 promotes growth and metastasis via activation of MAPK/AKT signaling in colorectal cancer. J. Cancer. 2019;10:874-84

27. Xia ZK, Yuan YC, Yin N, Yin BL, Tan ZP, Hu YR. Nonmuscle myosin IIA is associated with poor prognosis of esophageal squamous cancer. Dis. Esophagus. 2012;25:427-36.

28. Xu Z, Li P, Wei D, Wang Z, Bao Y, Sun J, et al. NMMHC-IIA-dependent nuclear location of CXCR4 promotes migration and invasion in renal cell carcinoma. Oncol. Rep. 2016;36:2681-2688.

29. Katono K, Sato Y, Jiang SX, Kobayashi M, Nagashio R, Ryuge S, et al. Prognostic significance of MYH9 expression in resected non-small cell lung cancer. PLoS One. 2015;10:e0121460.

30. Arora S, Saha S, Roy S, Das M, Jana SS, Ta M. Role of nonmuscle myosin II in migration of wharton's Jelly-Derived mesenchymal stem cells. Stem Cells Dev. 2015;24:2065-2077.

31. Zhao B, Qi Z, Li Y, Wang C, Fu W, Chen YG. The non-muscle-myosin-II heavy chain Myh9 mediates colitis-induced epithelium injury by restricting Lgr5+ stem cells. Nat. Commun. 2015;6:7166

32. Chatterjee S, Sil PC. Targeting the crosstalks of Wnt pathway with Hedgehog and Notch for cancer therapy. Pharm. Res. 2019;142:251-261.

33. Sari IN, Phi LTH, Jun N, Wijaya YT, Lee S, Kwon HY. Hedgehog Signaling in Cancer: A Prospective Therapeutic Target for Eradicating Cancer Stem Cells. Cells. 2018;7:208.

34. Zhu P, Fan Z. Cancer stem cells and tumorigenesis. Biophys. Rep. 2018;4:178-188.

35. Feng L, Wu JB, Yi FM. Isolation and phenotypic characterization of cancer stemlike side population cells in colon cancer. Mol. Med Rep. 2015;12:3531-3536.

36. Han ME, Jeon TY, Hwang SH, Lee YS, Kim HJ, Shim HE, et al. Cancer spheres from gastric cancer patients provide an ideal model system for cancer stem cell research. Cell Mol. Life Sci. 2011;68:3589-3605. 
10

37. Haraguchi N, Utsunomiya T, Inoue H, Tanaka F, Mimori K, Barnard GF, et al. Characterization of a side population of cancer cells from human gastrointestinal system. Stem Cells. 2006;24:506-513.

38. Hong KJ, Wu DC, Cheng KH, Chen LT, Hung WC. RECK inhibits stemness gene expression and tumorigenicity of gastric cancer cells by suppressing ADAMmediated Notch1 activation. J. Cell Physiol. 2014;229:191-201.

39. Coaxum SD, Tiedeken J, Garrett-Mayer E, Myers J, Rosenzweig SA, Neskey DM. The tumor suppressor capability of p53 is dependent on non-muscle myosin IIA function in head and neck cancer. Oncotarget. 2017;8:22991-23007.

40. Ye G, Huang K, Yu J, Zhao L, Zhu X, Yang Q, et al. MicroRNA-647 Targets SRFMYH9 Axis to Suppress Invasion and Metastasis of Gastric Cancer. Theranostics. 2017;7:3338-3353.

41. Yu M, Wang J, Zhu Z, Hu C, Ma Q, Li X, et al. Prognostic impact of MYH9 expression on patients with acute myeloid leukemia. Oncotarget. 2017;8:156-163.

\section{ACKNOWLEDGEMENTS}

This research was supported by CAMS Innovation Fund for Medical Sciences (Grant Number: 2016-12M-3-005), National Natural Science Foundation of China (Grant Number: 82073278), the Independent Issue of State Key Laboratory of Molecular Oncology (Grant Number: SKLMO-2021-17), Beijing Municipal Health Commission (Grant Number: BMC2018-4 and BMC2019-9), Natural Science Foundation of Beijing JiShuiTan Hospital (Grant Number: ZR-201906).

\section{AUTHOR CONTRINUTIONS:}

M.C.: Methodology, Formal analysis, Writing-Original draft preparation; L.S.: Software, Investigation; L.Y.: Validation, Formal analysis; J.L.: Data Curation; L.S.: Resources, Data Curation; Z.Y.: Validation; Writing - Review \& Editing; X.S.: Conceptualization, Project administration, Writing-Review \& Editing; Y.R.: Conceptualization, Supervision. All authors read and approved the final paper.

\section{COMPETING INTERESTS}

The authors no competing interest.

\section{ADDITIONAL INFORMATION}

Supplementary information The online version contains supplementary material available at https://doi.org/10.1038/s41420-021-00681-z.

Correspondence and requests for materials should be addressed to Xiong Shu or YuLiang Ran.

Reprints and permission information is available at http://www.nature.com/ reprints

Publisher's note Springer Nature remains neutral with regard to jurisdictional claims in published maps and institutional affiliations.

(i) Open Access This article is licensed under a Creative Commons Attribution 4.0 International License, which permits use, sharing, adaptation, distribution and reproduction in any medium or format, as long as you give appropriate credit to the original author(s) and the source, provide a link to the Creative Commons license, and indicate if changes were made. The images or other third party material in this article are included in the article's Creative Commons license, unless indicated otherwise in a credit line to the material. If material is not included in the article's Creative Commons license and your intended use is not permitted by statutory regulation or exceeds the permitted use, you will need to obtain permission directly from the copyright holder. To view a copy of this license, visit http://creativecommons. org/licenses/by/4.0/.

(c) The Author(s) 2021 